\title{
Performance Evaluation of Non linear Companding Transform MIMO-OFDM
}

\author{
K.Srinivasarao, \\ Electronics and Communications, \\ G. V. P. College of Engineering for Women, \\ Visakhapatnam, A.P., India
}

\author{
B.Prabakararao, Ph.D \\ Electronics and Communications, \\ J. N. T University, \\ Kakinada, A.P., India
}

\begin{abstract}
In this paper, we deal with the challenges regarding the provision of Inter Carrier interference (ICI) and Peak-to-Average Power Ratio (PAPR) for the performance evaluation of Multiple-Input and Multiple-Output Orthogonal Frequency Division Multiplexing (MIMO-OFDM). We propose a Non-linear Companding Technique (NCT) based on the inverse hyperbolic cosine function for PAPR reduction in OFDM symbol. Orthogonality among subcarriers is maintained and PAPR performance is achieved simultaneously by combing NCT and ICI cancellation techniques. Carrier to interference ratio (CIR) remains unchanged and extra Side Information (SI) is not required to recover the original signal as in the case of PTS and SLM techniques. Simulation results support BER improvement over existing methods in Additive White Gaussian Noise channel.
\end{abstract}

\section{Keywords}

Multiple-Input and Multiple-Output Orthogonal Frequency Division Multiplexing (MIMO-OFDM), Inter Carrier interference (ICI), Peak-to-Average power ratio (PAPR), and Non linear Companding Transform (NCT). Carrier to Interference ratio (CIR), Bit Error Rate (BER).

\section{INTRODUCTION}

Robustness of Orthogonal Frequency Multiplexing (OFDM) systems in multipath environments highlights the substantial potential of MIMO-OFDM systems [1-3]. Very high data rates achieved by MIMO-OFDM systems have been adopted in $3^{\text {rd }}$ generation partnership project long term evaluation (3GPP-LTE) [4], and IEEE 802.16 (Wi-MAX) [5]. Due to the mismatch in local oscillator frequencies of OFDM transmitter and receiver and the Doppler spread caused by user mobility the orthogonality between subcarriers is affected and creates Inter Carrier Interference.

ICI is to be minimized for improvement of CIR and BER performance in OFDM. Different approaches have been proposed in the literature to eliminate the effect of ICI, that include carrier frequency offset (CFO) estimation and removal [7-10] and frequency domain equalization scheme [11] involving highly complex matrix inversion. To take the advantage of frequency diversity, new ICI cancellation scheme [12] repeats the data symbols on two symmetrically located subcarriers.
In addition, OFDM signals exhibit high Peak-to-Average Power Ratio (PAPR), causing MIMO-OFDM signals transmitted from different antennas to exhibit a prohibitively high PAPR [6] if reduction measures are not implemented. It results in overall performance degradation.

Promising approach called non-linear companding transform (NCT) was proposed to overcome the issue of Out of Band Interference caused by PAPR increase. This method [13] employs the well known $\mu$-law companding and significantly outperforms the clipping. However, its average output power increases after such a logarithmic-based compression which may make it oversensitive to the High Power Amplifier (HPA). Recently non linear companding methods were proposed in [1415] but companding complexity is more in these methods.

Simultaneous reduction of ICI and PAPR is desirable for efficient performance of MIMO-OFDM systems in mobile communications systems.

To reduce the complexity in [14-15] we proposed a new non linear companding method whose companding function is defined by an inverse hyperbolic cosine function with an inflexion point. In the proposed method, the power distribution of the companded signal can be reallocated more reasonably while maintaining average power level, so that significant PAPR reduction as well as improved bit error rate (BER) performance can be achieved simultaneously. By adjusting the variable companding parameters, it allows more flexibility and freedom in the companding form so that an efficient trade-off between PAPR and BER performance can be achieved. Theoretical analysis and simulations confirm the effectiveness of the scheme.

The remainder of this paper is organized as follows: Section 2 gives a brief on MIMO-OFDM system model. ICI cancellation scheme and proposed companding transform for PAPR reduction are described in section 3. Section 4 is devoted to the analysis of simulation results and section 5 gives concluding remarks.

\section{MIMO-OFDM System model}

Consider an STBC-OFDM system with $\mathrm{M}_{\mathrm{T}}$ transmitter and $\mathrm{M}_{\mathrm{R}}$ receiver antennae with full rate Alamouti spatial coding, is as shown in Fig.1. The non-linear companding transform is applied in two stages namely ICI-SC and IFFT\&NCT. In ICI-SC the algorithm for CIR is estimated and in IFFT\&NCT Companding algorithm for PAPR reduction is verified. So overall evaluation in this combination is analyzed in terms BER performance. 


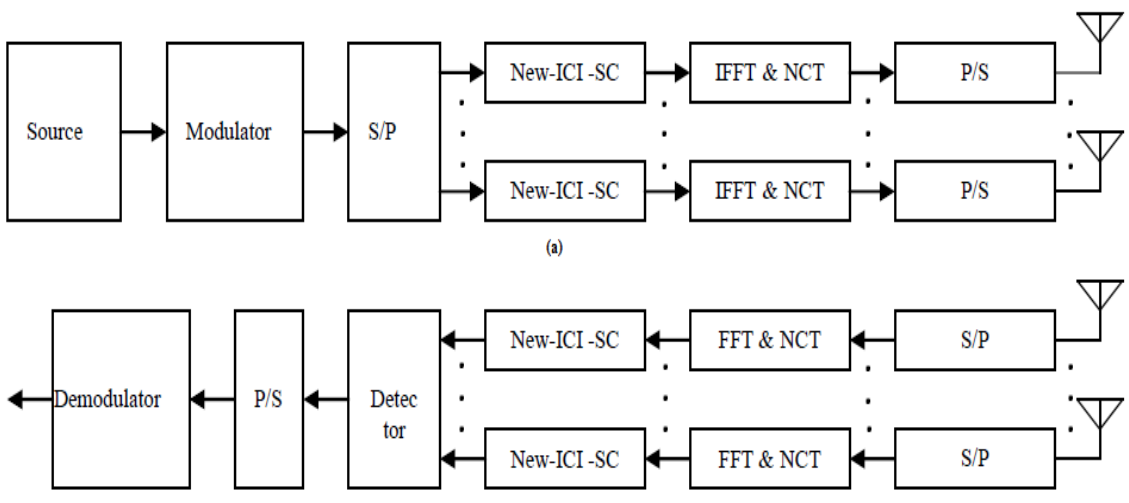

Figure 1.Block diagram of the MIMO-OFDM system (a) Transmitter (b) Receiver.

2 X 2 MIMO Alamouti coding can be expressed as (1)

$$
G=\left[\begin{array}{cc}
X(2 v) & X(2 v+1) \\
-X^{*}(2 v+1) & X^{*}(2 v)
\end{array}\right]
$$

where $\quad v=0,1,2, \ldots, N_{C} / 2-1$.

Symbols $X(1)$ and $X(2)$ are transmitted over antennas 1 and 2 on tone ' $n$ ' and symbols $-\mathrm{X}(2)^{*}$ and $\mathrm{X}(1)^{*}$ are transmitted over antennas 1 and 2 on tone ' $n+1$ '.

At the transmitter side, a serial bit stream is mapped to a symbol stream by a digital modulator. This serial symbol stream is converted into parallel sub streams. New ICI-self cancellation modulation is performed at this stage. Then, the OFDM modulation is implemented by performing inverse DFT (IFFT). Each transmit antenna sends independent OFDM symbols, let $X_{P}(k)$ denote the information sent by transmit antenna ' $\mathrm{P}$ ' at the subcarrier $\mathrm{k}$. The OFDM symbols transmitted by $\mathrm{M}_{\mathrm{T}}$ transmit antennas can be represented as.

$$
X=\left[X_{1}, X_{2}, \ldots, X_{p}, \ldots, X_{M_{T}}\right]^{T}
$$

where $X_{P}=\left[X_{P}(0), X_{P}(1), \ldots, X_{P}(N-1)\right]^{T}$ is the OFDM symbol transmitted from the $p^{\text {th }}$ transmit antenna, and $\mathrm{N}$ is the number of subcarrier for OFDM symbol. After performing IFFT on each transmit antenna, the time domain modulated signal on the $p^{\text {th }}$ transmit antenna can be expressed as $x_{P}=F^{H} \cdot X_{P}=\left[x_{p}(0), \ldots\right.$, $\left.x_{p}(N-1)\right]^{T}$, where $F$ is the [NXN] DFT matrix with its elements at row $n$ and column $k$, which is defined as $W_{n, k}=e^{(-j 2 \pi n k / N)}$ for $\mathrm{n}$, $k=0,1, \ldots, \mathrm{N}-1$.

To avoid Intercarrier interference (ICI) new ICI modulation scheme is implemented by repeating the data symbols on two symmetrically located subcarriers, and a cyclic prefix is inserted in each OFDM symbol prior to transmission as guard interval (GI) between OFDM symbols. Finally the symbol streams are converted from a parallel-to-serial form and allocated to corresponding transmitter for transmission.

The received signal at the $q^{\text {th }}$ receiver antenna and time ' $\mathrm{n}$ ' (after GI is removed from OFDM symbols) can be represented as:

$$
\begin{aligned}
& r_{q}(n)=\sum_{p=1}^{M_{T}}\left(h_{p, q}(l, n) \otimes x_{p}(n)\right)+w_{q}(n) \\
& =\sum_{p=1}^{M_{T}} \sum_{l=1}^{L-1} h_{p, q}(l, n) x_{p}(n-l)+w_{q}(n)
\end{aligned}
$$

where $\otimes$ is cyclic convolution, $w_{q}(n)$ is additive white Gaussian noise (AWGN), and $h_{p, q}(l, n)$ is impulse response of the $l^{\text {th }}$ channel tap between the $p^{\text {th }}$ transmit antenna and the $q^{\text {th }}$ receive antenna at time ' $n$ '.

After performing DFT on the received signal (3), the symbol for the $\mathrm{q}^{\text {th }}$ receive antenna and $k^{\text {th }}$ subcarrier can be represented as:

$R_{q}(K)=\sum_{p=1}^{M_{T}} \sum_{m=0}^{N-1} \sum_{l=0}^{L-1} H_{l}^{p, q}(k-m) w_{l, m} x_{p}(m)+W_{q}(K)$

where $W_{q}(K)$ is the DFT of noise and $H_{l}^{p, q}(\mathrm{~K})$ denotes the DFT of time varying frequency selective channel $h_{p, q}(1, \mathrm{n})$ i.e.,

$H_{l}^{p, q}(K)=\sum_{n=0}^{N-1} h_{p, q}(l, n) e^{\frac{-j 2 \pi n k}{N}}$

$R_{q}(K)$ can be further expressed as summation of the desired signal and ICI component

$R_{q}(K)=\underbrace{\sum_{p=1}^{M_{T}} \sum_{l=0}^{L-1} H_{l}^{p, q}(0) w_{l, k} x_{p}(k)}_{\text {Desired Signal }}+$

$\sum_{p=1}^{M_{T}} \sum_{l=0}^{N-1} \sum_{l=0}^{L-1} H_{l}^{p, q}(k-l) w_{l, m} X_{p}(m)$

$\underbrace{\sum_{\substack{p=k \\ m \neq k}}}_{\text {ICIcomponent }}+W_{q}(K)$

The received signal for all $\mathrm{M}_{\mathrm{R}}$ receive antennas can be represented as

$R=H \cdot X+W$

where $R=\left[R_{l}, \ldots, R_{M R}\right]^{T}$, and $R_{q}=\left[R_{q}(0), \ldots, R_{q}(N-1)\right]^{T}$ is the received signal for the $q^{\text {th }}$ receiver antenna, $w=\left[w_{l}, \ldots, w_{M R}\right]^{T}$ is AWGN noise, and $\mathrm{H}$ is the effective channel matrix in the frequency domain, which is defined as,

$H=\left[\begin{array}{cc}H_{1,1} & H_{2,1} \cdots H_{M T, 1} \\ H_{1,2} & H_{2,2} \cdots H_{M T, 2} \\ & \ddots \\ H_{1, M R} & H_{2, M R} \cdots H_{M T, M R}\end{array}\right]$

here the $(m, n)^{\text {th }}$ element of matrix $H_{p, q}$ is defined as $\alpha_{m, n}^{p, q}$ and defined as

$\alpha_{m, n}^{p, q}=\sum_{l=0}^{L-1} H_{l}^{p, q}(n-m) w_{l, m} ; \mathrm{n} \geq 0 ; \mathrm{m} \leq N-1$

The carrier frequency offset, $\Delta \varepsilon$, gives additional phase factor of $\mathrm{e}^{\mathrm{j} 2 \pi \Delta \varepsilon \mathrm{t}}=\mathrm{e}^{\mathrm{j} 2 \pi \varepsilon \mathrm{ft}}=\mathrm{e}^{\mathrm{j} 2 \pi \varepsilon \mathrm{n} / \mathrm{N}}$ to the received signal, where $\mathrm{f}$ is the sub-carrier spacing, $\varepsilon$ is the normalized $\mathrm{CFO}$ by $\mathrm{f}$ and $\mathrm{n}=0,1, \cdots \mathrm{N}-1$.

\section{NEW ICI SELF CANCELLATION}

Generic ICI self cancellation based OFDM system experiences same channel frequency response on two adjacent sub-carriers and therefore cannot achieve much frequency diversity on two adjacent sub-carriers. Therefore 
modified ICI self cancellation scheme is used to improve the CIR performance over frequency selective fading channel. In this scheme data symbols are repeated on two symmetrically located sub-carriers using polynomial

$X(N-1)=-X(0), X(N-2)=-X(1) \ldots . X(N-K)=-X(K)$

The expression of discrete time domain OFDM signal using above expression can be written as

$x(\mathrm{n})=\frac{1}{\sqrt{\mathrm{N}}} \sum_{\mathrm{k}=0}^{\frac{\mathrm{N}}{2}-1} X_{k}\left\{\exp \left(\frac{\mathrm{j} 2 \mathrm{nkn}}{\mathrm{N}}\right)-\exp \left(\frac{\mathrm{j} 2 \mathrm{n}(\mathrm{N}-1-\mathrm{k}) \mathrm{n}}{\mathrm{N}}\right)\right\}$

$x(n)=\frac{2 j}{\sqrt{N}} \exp \left(\frac{-j n n}{N}\right) \sum_{k=0}^{\frac{N}{2}-1} X_{k} \sin \left(\frac{j 2 n\left(k+\frac{1}{2}\right) n}{N}\right)$

The expression of CIR performance for New ICI self cancellation scheme is

$C_{N S C}=\frac{|2 S(0)|^{2}}{\sum_{l=1}^{\frac{N}{2}-1}|S(l)-S(N-1-l)-S(l-N+1)+S(-l)|^{2}}$

\subsection{Peak-to-average-power ratio (PAPR)}

PAPR is the ratio of peak power to average power of OFDM signal. For STBC MIMO-OFDM (1) signal x [n] of $i^{\text {th }}$ antenna PAPR is given by

$\operatorname{PAPR}(\mathrm{dB})=10 \log _{10}\left(\frac{\max \left\{|x[n]|^{2}\right\}}{E\left\{|x[n]|^{2}\right\}}\right)$

where E[.] denotes expectation. If a discrete-time STBC MIMOOFDM signal $(\mathrm{x}[\mathrm{n}])$ is over sampled by a factor $l \leq 4$, it is sufficient for capturing the continuous-time peaks, and to prevent large analog "peak re-growth" at the D/A converter.

\subsection{Proposed companding transform for PAPR reduction}

This subsection derives the formulas of the new transform, whose companding function is defined by an inverse hyperbolic sine function with an inflexion point. Using this approach, the amplitude or power of the transmitted signal can be reallocated more reasonably and flexibly. In addition, it has the advantage of maintaining constant average output power levels. Thus, the sensitivity of companding operation to the non linearity of HPA can be partially averted. In OFDM system, the companded signal $y=\left[y_{0}, y 1 \ldots . y_{L N-1}\right]^{T}$ is given by

$Y n=C\left(x_{n}\right) \quad n=0,1, \ldots . L N-1$

where $\mathrm{X}_{\mathrm{n}}$ is the original OFDM signal, and $C($. ) represents the companding function, which is a strictly monotonic increasing function and changes only the amplitude of $x_{n}$. The inverse of the companding function is the de-companding function, i.e. $\mathrm{C}^{-1}(\mathrm{x})$. Suppose $\mathrm{cA}_{\mathrm{x}}(0 \leq \mathrm{c} \leq 1)$ is the inflexion point of $C(x)$ where $\mathrm{A}_{\mathrm{x}}$ is the maximum input amplitude.

$A_{x}=\max _{\mathrm{n} \in[0, \mathrm{LN}-1]}\left\{\left|x_{\mathrm{n}}\right|\right\}$

The proposed companding function is defined as follows

$C(x)= \begin{cases}\operatorname{sgn}(x) \cdot \gamma \cdot \operatorname{arcosh}(K|x|), & |x| \leq c A_{x} \\ \operatorname{sgn}(x) \cdot \gamma \cdot \operatorname{arcosh}\left(K c A_{x}\right), & |x|>c A_{x}\end{cases}$

where $\operatorname{sgn}($.$) is the sign function, and the inverse hyperbolic$ cosine function $\operatorname{arcosh}($.$) is given by$

$\operatorname{arcosh}(x)=\ln \left(x+\sqrt{x^{2}-1}\right)$

The parameter $\gamma$ is used to adjust the average output power level, and $K$ is the factor specifying the degree of companding form. Under constraint with respect to the average power before and after the companding operation, we let
$E\left\{\left|y_{n}\right|^{2}\right\}=E\left\{\left|x_{n}\right|^{2}\right\}=\int_{0}^{\infty} x^{2} f_{\left|x_{n}\right|}(x) d x$

By solving the above equation we have,

$\gamma=\frac{\sigma}{\sqrt{E\left\{\operatorname{arcosh}^{2}{ }_{n \subset\left\{\left|x_{n}\right| \leq c A_{x}\right\}}\left(k\left|x_{n}\right|\right) \cup \operatorname{acosh}^{2}{ }_{n \subset\left\{\left|x_{n}\right|>c A_{x}\right\}}\right\}}}$

When $K$ is chosen, $\gamma$ can be also determined. At the receiver, the companded signal can be correctly recovered by the corresponding de-companding function as follows.

$C^{-1}(x)=\operatorname{sgn}(x) \cdot \frac{1}{K} \cdot \cosh \left(\frac{|x|}{\gamma}\right)$,

where $\cosh (x)=\frac{e^{x}+e^{-x}}{2}$ is the hyperbolic sine function.

Further the target CDF of the companded signal is obtained as $F_{\left|y_{n}\right|}(x)=\operatorname{Prob}\left\{\left|y_{n}\right| \leq x\right\}=\operatorname{Prob}\left\{C\left(x_{n}\right) \leq x\right\}$

$=$

$\left\{\begin{array}{cl}0, & x<0 \\ 1-\exp \left(-\frac{\cosh ^{2}(x / \gamma)}{K^{2} \sigma^{2}}\right), & 0 \leq x<\gamma \cdot \operatorname{arsinhKc} A_{x} \\ 1, & x \geq \gamma \cdot \operatorname{arsinhKc} A_{x}\end{array}\right.$

In a practical OFDM system, the proposed scheme is implemented via the pre-computed look-up tables for real time application. The transfer curves of (17) with various parameters are depicted in Figure 2.

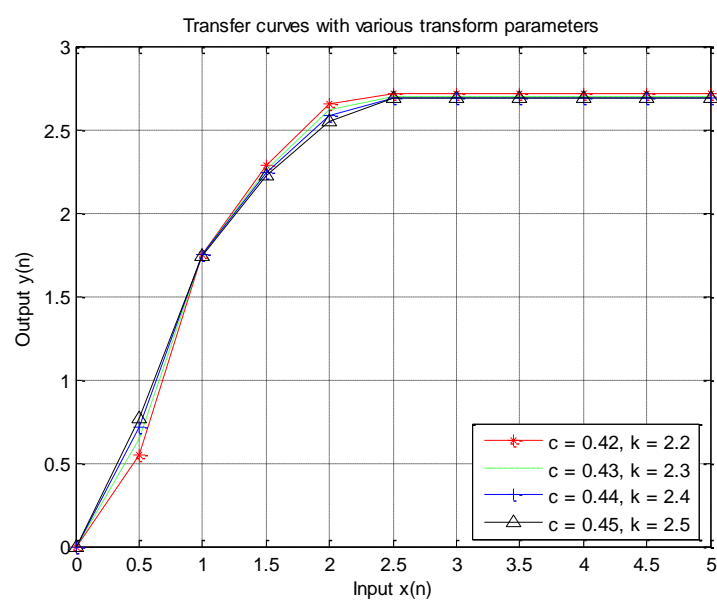

Figure 2.Transfer curves with various transform parameters.

It can be seen from Figure 2 that this method compresses large signals while partially enlarging small ones simultaneously. It has the advantage of maintaining a constant average power level in the transform. As a result, not only is the PAPR reduced more effectively, but the immunity of small signals from the channel noise also is achieved.

\section{ANALYSIS OF THE SIMULATION RESULTS}

The simulation results of the proposed system combination of ICI reduction and companded STBC MIMO-OFDM are presented in the following figures. With $\mathrm{N}=512$ subcarriers and 16-QAM modulation over AWGN channel are considered for analysis of the proposed system in MATLAB.

Figure 3 shows the CIR of New ICI Cancellation scheme achieved is high. From the graphs it is clear that New-Self Cancellation scheme exhibits high CIR over existing methods. 


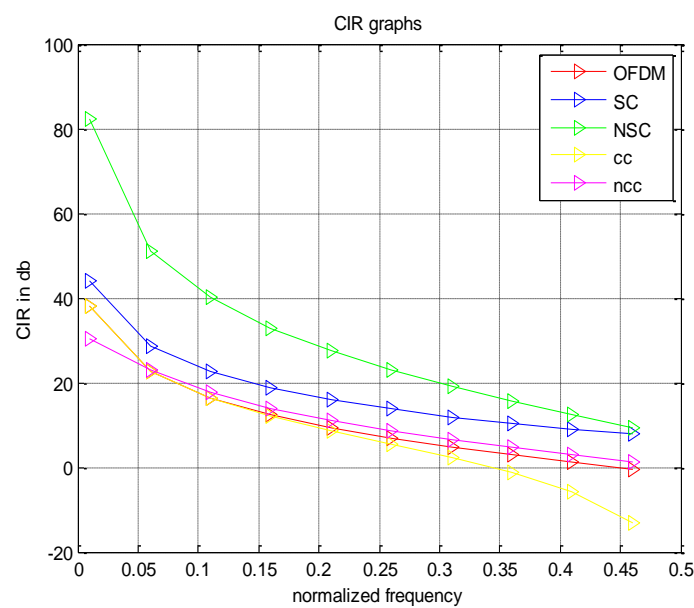

Figure 3.CIR performance of various ICI cancellation schemes

Figure 4 depicts that CCDF based comparison of PAPR of the proposed system for various parameters are compared with original OFDM. At clip rate of $10^{-3}$, the PAPR gains are $4.5 \mathrm{~dB}$ over OFDM.

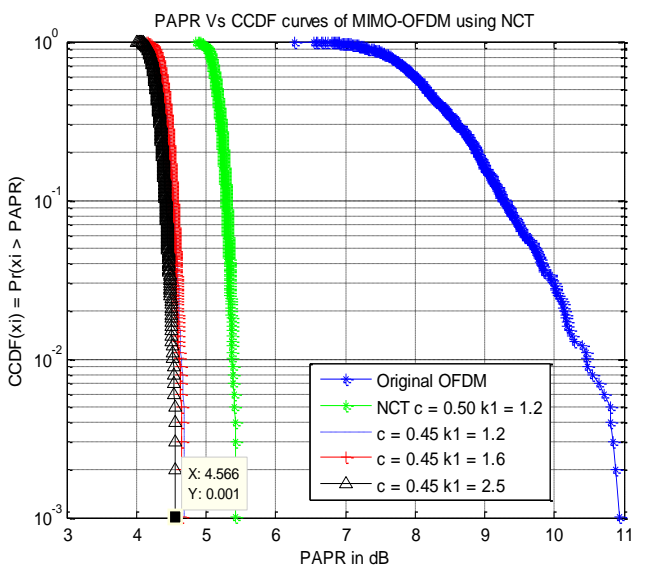

Figure 4.CCDF Vs PAPR curves of various $C$ and $K$ values.

Figure 5 depicts the combination of new ICI cancellation and proposed Companding have been performed better in terms BER performance. It is clear that sammler BER value is aciheved for lesser $\mathrm{Eb} / \mathrm{No}$ than original OFDM.

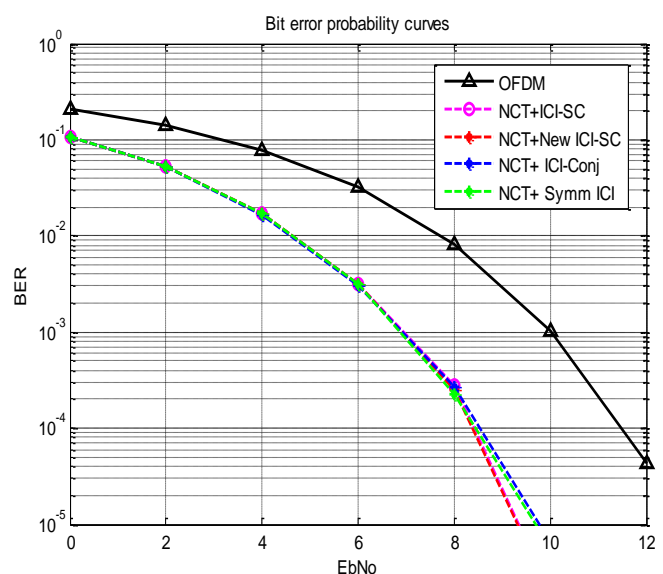

Figure 5.BER graphs of combination of ICI and NCT.
Figure 6 shows that power level has been decrased after applying the proposed companding on OFDM.

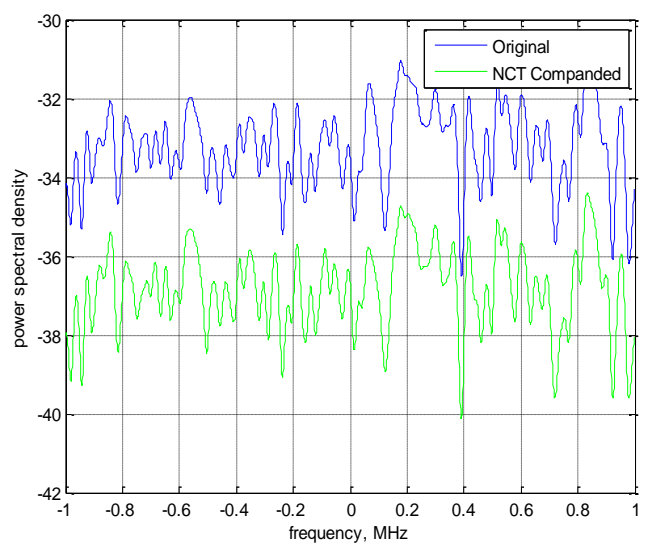

Figure 6.PSD curves of original OFDM and NCT companded OFDM.

\section{CONCLUSIONS}

In this paper, a PAPR reduction method for STBC MIMOOFDM Systems using the inverse hyperbolic sine function is proposed and evaluated. In this method large signals are compressed and small signals are partially enhanced simultaneously resulting in uniform power distribution of the companded signals. Power is allocated more reasonably among the signals; significant PAPR reduction is achieved with less companding distortion. The proposed PAPR reduction scheme with New ICI self cancellation is combined to improve the CIR and BER performance for performance evaluation of MIMOOFDM. From the simulation results the proposed method has PAPR gain of $4.5 \mathrm{~dB}$ and improved BER performance over conventional OFDM systems. The proposed system requires no side information as compared to PTS and SLM methods.

\section{ACKNOWLEDGMENTS}

The authors sincerely thank Prof. G.T Rao, E.C.E Department, G.V.P College of Engineering (Autonomous) for his immense support and valuable suggestions.

\section{REFERENCES}

[1] MK Ozdemir, H Arslan, Channel estimation for wireless OFDM systems. IEEE Commun. Surv. Tutor. 9(2), 18-48 (2007)

[2] M Sandell, J Coon, Near-optimal training sequences for MIMO OFDM systems with nulled subcarriers. in Proc. IEEE Global Telecommunications Conference GLOBECOM '05, vol. 4, 2243-2249, (2005)

[3] Q Huang, M Ghogho, S Freear, Pilot design for MIMO OFDM systems with virtual carriers. IEEE Trans. Signal Process. 57(5), 2024-2029 (2009)

[4] Physical Layer Aspects for Evolved UTRA, Std. TR 25.814, Rev. 7.1.0, Sep. 2006.

[5] IEEE Standard for Local and Metropolitan Area Networks Part 16: Air Interface for Fixed and Mobile Broadband Wireless Access Systems Amendment 2: Physical and Medium Access Control Layers for Combined Fixed and Mobile Operation in Licensed Bands and Corrigendum 1, IEEE Std. 802.16e-2005, 2006.

[6] B Rihawi, Y Louet, S Zabre, PAPR reduction scheme with SOCP for MIMO-OFDM. in Proc. Int. Conf. Wireless 
Communications, Networking and Mobile Computing WiCom 2007, 271-274, (2007).

[7] van de Beek, J. J., Sandell, M., \& Borjesson, P. O. (1997). ML estimation of time and frequency offset in OFDM systems. IEEE Transactions on Signal Processing, 45(7), $1800-1805$.

[8] Tureli, U., Kivanc, D., \& Liu, H. (2001). Experimental and analytical studies on a high-resolution OFDM carrier frequency offset estimator. IEEE Transactions on Vehicular Technology, 50(2), 629-643.

[9] Fernandez-Getino Garcia, M. J., Edfors, O., \& PaezBorrallo, J. M. (2001). Frequency offset correction for coherent OFDM in wireless systems. IEEE Transactions on Consumer Electronics, 47(1), 187-193.

[10] Luise, M., Marselli, M., \& Reggiannini, R. (2002). Lowcomplexity blind carrier frequency recovery for OFDM signals over frequency-selective radio channels. IEEE Transactions on Communications, 50(7), 1182-1188.

[11] Ahn, J.,\&Lee, H. S. (1993). Frequency domain equalization of OFDM signals over frequency nonselective Rayleigh fading channels. IEEE Electronics Letters, 29(16), 14761477.

[12] Wang, C. L., \& Huang, Y. C. (2010). Inter Carrier interference cancellation using general phase rotated conjugate transmission for OFDM systems. IEEE Transactions on Communications, 58(3), 812-819.

[13] X. B. Wang, T. T. Tjhung, and C. S. Ng, "Reduction of peak-to-average power ratio of OFDM system using a companding technique," IEEE Trans. Broadcast., vol. 45, no. 3, pp. 303-307, Sep. 1999.
[14] Shiann-Shiun Jeng, Member, IEEE, and Jia-Ming Chen, Student Member, IEEE," Efficient PAPR Reduction in OFDM Systems Based on a Companding Technique with Trapezium istribution", IEEE TRANSACTIONS ON BROADCASTING, VOL. 57, NO. 2, JUNE 2011

[15] Yong Wang, Jianhua Ge, Lihua Wang, Jing Li, "Reduction of PAPR of OFDM Signals Using Nonlinear Companding Transform” Springer Science+Business Media, LLC. 2012

K. Srinivasa Rao received his B. Tech in Electronics and Communication Engineering from Nagarjuna University, Guntur and M. Tech in Instrumentation and Control Systems from J.N.T University College of Engineering. Kakinada, he has 10 years of teaching experience and is a Sr. Assistant Professor of Electronics and Communication Engineering, G.V.P. College of Engineering for Women, Visakhapatnam. His research interests include Wireless Communications and Signal processing. He is currently perusing Ph.D. from J.N.T.U. Kakinada and is a Life Member of IETE.

Dr.B.Prabhakara Rao received his B.Tech in Electronics and Communications Engineering. M.Tech degree in Electronics and Communication Systems from S. V. University, Tirupati in 1979, 1981 respectively and received the Ph.D from IISc, Bangalore in 1995. Dr.B.Prabhakara Rao has more than 32 years of experience in teaching and Research. $\mathrm{He}$ is an expert in Signal Processing \& Communications. He has produced 17 PhDs and presently 20 scholars are perusing their Ph.D. program under his guidance. He held different positions in his career like Head of the Department, Vice Principal, in JNTU College of Engineering etc., and currently he is the Rector of the JNT University. He has published more than 175 technical papers in the reputed National and International journals and conferences. His research interests are coding theory, Information theory and Signal processing with applications to wireless communications. 